\title{
Effects of Transcranial Direct Current Stimulation on Cycling Time Trial Perfor- mance and Prefrontal Cortex Activation
}

\author{
Abi Auten ${ }^{1}$, Kristina Cavey ${ }^{1}$, Jacob Reed ${ }^{1}$, Forrest Dolgener ${ }^{1}$ and Terence Moriarty 1,* \\ 1 Department of Kinesiology, University of Northern Iowa, Cedar Falls, IA 50614, USA; autena@uni.edu \\ (A.A.); caveyk@uni.edu (K.C.); jacob.reed@uni.edu (J.R.); forrest.dolgener@uni.edu (F.D.) \\ * Correspondence: terence.moriarty@uni.edu; Tel.: +1 319-273-6866
}

\begin{abstract}
Background: Transcranial direct current stimulation (tDCS) is a neuromodulatory technique that delivers low levels of a constant current via scalp electrodes to specifically targeted areas of the brain. The effects of tDCS on whole-body exercise performance has been of interest in recent literature. The purpose of the current investigation was to investigate if tDCS, administered via Halo Sport, influences time trial performance in trained cyclists, and if changes in exercise performance are associated with prefrontal cortex (PFC) activation and/or muscle oxygenation $\left(\mathrm{SmO}_{2}\right)$. Methods: Twelve recreationally trained cyclists volunteered to participate in two 10-kilometer time trials following 20 minutes of $\mathrm{tDCS}$ or a sham condition. Results: T-tests showed there was no significant difference in performance (time to completion) or physiological measures (BLa, $\mathrm{HR}, \mathrm{SmO}_{2}, \mathrm{PFC}$ oxygenation) between the Halo and sham conditions. Conclusions: These results indicate that the application of tDCS via Halo Sport does not induce changes in exercise performance or related physiological parameters during a 10-kilometer cycling time trial.
\end{abstract}

Keywords: tDCS; cycling time trial; exercise performance; prefrontal cortex; muscle oxygenation

\section{Introduction}

The limiting factors of exercise performance have been the focus of many current and past exercise science research discussions [1-3]. During continuous submaximal exercise, the ability of the skeletal muscle fibers to contract and the excitability of motor neurons projecting from the central nervous system are significantly decreased [4]. To accommodate for this reduction in force or power output, the output signals from the motor cortex of the brain to the periphery (i.e., skeletal muscle) must be increased to generate enough force to maintain exercise intensity. Supraspinal fatigue can be described as a reduction in motor cortical neuronal drive or the lack of ability to generate output from the motor cortex, and in combination with peripheral factors (i.e., changes at or distal to the neuromuscular junction) can cause muscular fatigue. Previous research has suggested that the development of supraspinal fatigue is often coupled with changes in the excitability of the motor cortex [5].

A multitude of electrical and magnetic stimulation techniques have been developed and extensively researched to modulate excitability and output signals from the motor cortex [6]. Increasing the output from the motor cortex can delay the onset of supraspinal fatigue and likely improve exercise capacity [7]. Of these techniques, transcranial direct current stimulation (tDCS), and specifically its' effects on physical performance have gained much interest in current literature [8-10]. tDCS is a neuromodulatory intervention that delivers low levels of a constant current to specifically targeted areas of the brain, such as the motor cortex, which create excitability changes [10]. tDCS is a noninvasive stimulation technique that is safe, affordable and easy to administer. For these reasons, interest in $\mathrm{tDCS}^{\prime}$ potential ergogenic effects on physical performance has increased greatly. 
Excellence in sport performance not only requires a high level of physical capability, but also mental capability. Previous neuroimaging studies have reported that, during whole body aerobic exercise, the prefrontal cortex (PFC) increases neuronal activation (as measured by brain oxygenation via functional NIRS (fNIRS)) during submaximal aerobic exercise (up to approximately $80 \%$ of peak ability) but then decreases when intensity reaches a very hard or maximal effort [11]. More specifically, at very high or maximal intensities of exercise where competitive exercise may take place, there may be a shift in resources from areas required for cognitive function to areas required for motor control and maintenance of vital function (e.g., thermoregulation) [12]. This shift in resources may explain the reduction in PFC activation during very high intensities of aerobic exercise. While the effect of $\mathrm{tDCS}$ on changes in PFC activation during whole body aerobic exercise is not well understood, noninvasive fNIRS is a commonly used tool which would allow investigation into these changes. The advantages of fNIRS is that it is also portable and provides live feedback regarding physiological changes associated with brain activity. This may provide us with mechanistic insight into how tDCS can alter PFC activation during competitive exercise.

Therefore, the present study seeks to evaluate if tDCS (administered via Halo Sport) influences exercise time trial performance in recreationally trained cyclists. An additional objective is to explore if the change in exercise performance is associated with changes in PFC and muscle oxygenation. Results may provide important insights into the mechanisms of how tDCS influences both competitive exercise and brain and muscle activity, and further allow sports performance personnel to identify and utilize appropriate techniques to improve competitive performance.

\section{Materials and Methods}

\subsection{Study Design}

All participants served as their own control in a placebo-controlled, counterbalanced, crossover study with a repeated measures design. The participants visited the lab on three separate occasions. On the first occasion, participants completed an 8-minute submaximal bike test on a Monark stationary bike at the same time of the day as the $10 \mathrm{~km}$ time trials to eliminate any effects of circadian variations. On the two other occasions the participants performed a 10km cycling time trial following stimulation of a 20-min Halo Sport session either with (Halo) or without (sham) electrical current delivered to the primers. During stimulation, participants were seated, quiet, and relaxed while listening to music of their choosing. All trials were separated by at least 72 hours but no more than 10 days. Participants were wearing brain (fNIRS) and muscle (Moxy) oxygenation devices during both time trials. See Figure 1 for a detailed outline of the study design, including duration between exercise testing sessions. 


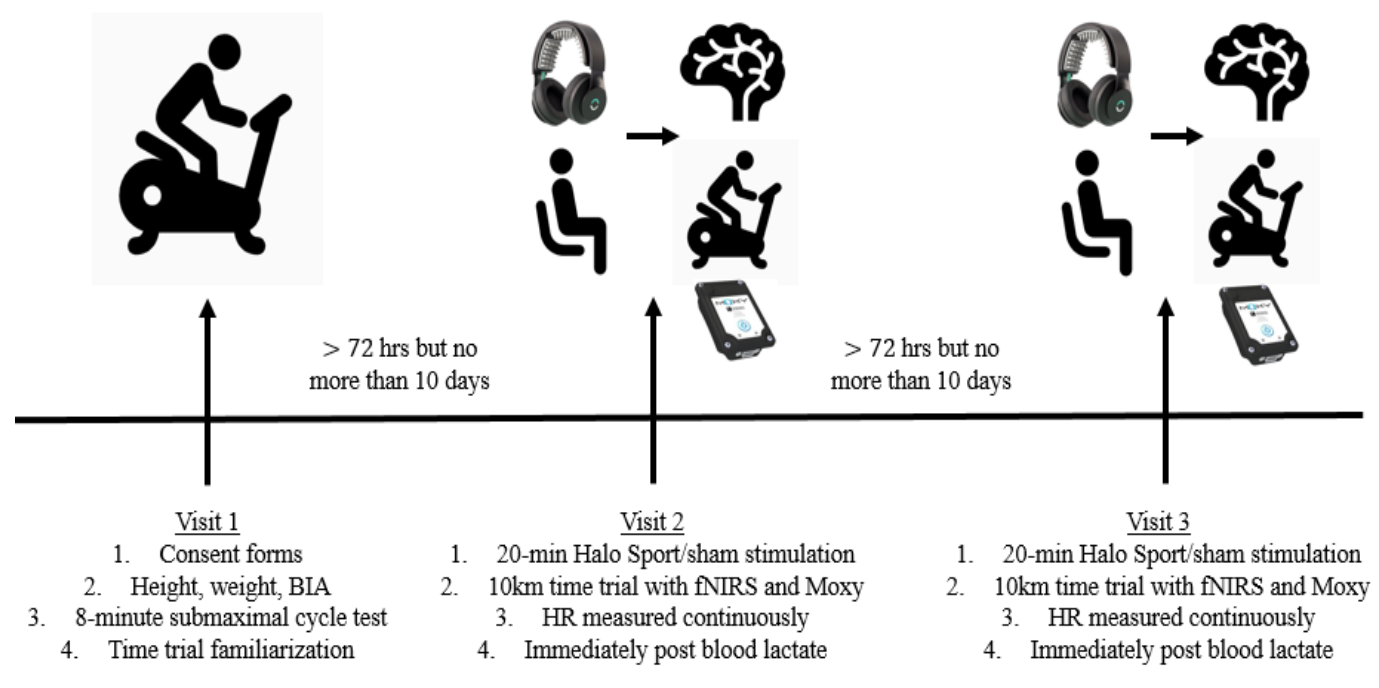

Figure 1. Detailed outline of the study.

\subsection{Participants}

All participants were informed of the study protocol approved by the University of Northern Iowa's Institutional Review Board (IRB) before obtaining written consent prior to participation in the study. Following IRB approval, recruitment flyers were posted. Twelve male, recreationally trained cyclists were recruited. All participants were active (no less than $30 \mathrm{~min} \cdot \mathrm{day}^{-1}, 3$ days week $^{-1}$, for at least 3 consecutive months) and were between the ages of 18 and 45 years. See Table 1 for subject demographics. All participants were administered a health history questionnaire which indicated that no subject had a history of musculoskeletal injuries, metabolic, cardiovascular or pulmonary disease, mental disorders/diseases, or were on medications during the study. Participants were asked to refrain from alcohol, caffeine, and physical activity for 24 hours before testing sessions. Participants were instructed to consume a meal of similar nutritional composition at the same time interval prior to all trials.

Table 1. Subject Demographics.

\begin{tabular}{c|c|c|c|c|c}
\hline Age (years) & Height $(\mathrm{cm})$ & Weight $(\mathrm{kg})$ & $\begin{array}{c}\text { BMI } \\
\left(\mathrm{kg} / \mathrm{m}^{2}\right)\end{array}$ & Body Fat $(\%)$ & $\begin{array}{c}\text { Estimated } \mathrm{VO}_{2} \text { max } \\
(\mathrm{ml} / \mathrm{kg} / \mathrm{min})\end{array}$ \\
\hline $25.5 \pm 7.8$ & $181.7 \pm 5.9$ & $85.9 \pm 12.3$ & $25.7 \pm 3.0$ & $15.3 \pm 7.0$ & $41.5 \pm 5.0$ \\
\hline
\end{tabular}

Data are expressed as means \pm standard deviations. $\mathrm{cm}=$ centimeters, $\mathrm{kg}=$ kilograms, $\mathrm{kg} / \mathrm{m}^{2}=$ kilograms per meter squared, $\mathrm{ml} / \mathrm{kg} / \mathrm{min}=$ milliliter per kilogram per minute.

\subsection{Anthropometric and Body Composition Measurements}

Prior to the first testing session, height $(\mathrm{cm})$ and body weight $(\mathrm{kg})$ was measured using a stadiometer and floor scale, respectively. Upon arrival for the first exercise testing trial (submaximal bike test), body fat percentage was also determined using bioelectrical impedance analysis (InBody 720).

\subsection{Exercise Testing and Screening}

All submaximal bike testing was performed on a Monark stationary bike (Ergomedic 828 E, Monark Exercise, Vansbro, Sweden) while wearing a Polar heart rate monitor (V800, Polar Electro Inc., Woodbury, NY, USA). The test began with a three-minute warm-up at 50 watts and then the intensity was increased to 150 watts and participants 
were asked to maintain $60 \mathrm{rpm}$ for the duration of 5 minutes. Heart rate was recorded at the cessation of the test and prediction equations were used to estimate $\mathrm{VO}_{2}$ max [13]. A $2 \mathrm{~km}$ familiarization of the time trial was also performed following the submaximal bike test to ensure participants understood the protocol and felt comfortable with the cycling intensities, and self-paced nature of the work intensity in the time trials. After a minimum of 72 hours following the submaximal bike test, participants returned to the exercise laboratory for one of the following two counter-balanced trials: tDCS (Halo) or no tDCS (sham).

\subsection{Time Trial Task}

Immediately after either tDCS via Halo or sham stimulation, participants performed a self-selected warm up. To assess endurance performance, participants performed a $10 \mathrm{~km}$ time-trial test on a VeloTron bike (VeloTron RacerMate, RacerMate Inc., Seattle, WA, USA). VeloTron bikes have a fully adjustable frame to fit multiple users and generates variable load ranges from 5 to 2000 watts, selected by the user.

After a five minute, self-selected warmup, participants started the $10 \mathrm{~km}$ cycling time trial under as consistent environmental conditions as possible. All time trials began from a standing start at a gear ratio of $53 \times 17$ and were completed using a virtual flat (i.e. zero gradient) course programed into the VeloTron software. Participants were asked to complete the time trial as quickly as possible, manipulating gearing as needed. Total time needed to complete the $10 \mathrm{~km}$ time trial was recorded from the VeloTron software. Overall RPE was also collected 10 minutes after exercise has ceased to provide an accurate quantification of session load (Uchida et al., 2014).

\subsection{Blood Lactate and Heart Rate}

A blood lactate [Bla-] measurement (Lactate Plus, Nova Biomedical, Waltham, WA, USA) was collected immediately post-exercise as a proxy of the intensity of that particular trial. All [Bla-] samples were collected at the earlobe using a lancing device, obtained in duplicate and averaged for analysis. Heart rate was also continuously measured throughout all trials using a Garmin heart rate monitor (HRM-Dual, Lenexa, KS, USA), which was integrated with the Moxy NIRS device.

\subsection{Halo Sport Procedures}

The Halo Sport headset itself is similar in appearance to an audio headset. The headset has foam electrodes (termed primers) which will be wetted prior to use to initiate the electrical current with the scalp. The headset will be positioned over the vertex of the head, with the primers lying across the top of the head, from ear to ear. The aim will be to stimulate both (left and right) sides of the motor cortex. The maximum energy output will be $2.2 \mathrm{~mA}$ and will be controlled by the Halo application on an iPhone or iPad.

Prior to exercise, participants were seated in a chair, in a resting state. The Halo Sport headset was correctly positioned on the head of participant and the electrical current will be turned to $2.0 \mathrm{~mA}$ over the course of 30 seconds. In the active Halo group, $2.0 \mathrm{~mA}$ was maintained for 20 minutes. In the sham group, intensity was ramped down after 30 seconds. This procedure is similar to previous studies done using Halo Sport [7, 14].

\subsection{Functional Infrared Spectroscopy Recording Procedures}

Prefrontal cortex (PFC) oxygenation was measured using a dual wavelength (760 and $850 \mathrm{~nm}$ ), portable fNIRS system (OctaMon, Artinis Medical Systems) during both time trial sessions. This device has been previously used to illustrate ecological validity during self-paced running [15]. Four LED optodes (transmitters) and one receiver were placed over the right and left PFC regions (RPFC and LPFC; 4x2 configuration). Optode placement is based on the modified international electroencephalogram 10/20 system [16]. The fNIRS cap was located 2 centimeters $(\mathrm{cm})$ above the nasion and centering on the Fpz location (distinctly depressed area directly between the eyes, just superior to the bridge of the nose). A source-detector distance of $3.5 \mathrm{~cm}$ was used, which is recommended as an 
optimal distance to detect cortical activity among adults [16, 17]. The signal sampling rate was $10 \mathrm{~Hz}$. In order to reduce possible disruptions in signals such as movement or heart rate, a moving 2-second average filter was applied to all raw data. Neural activity induces changes in blood flow to activated areas of the brain. When blood flow is increased in activated areas of the brain, local supply of oxygen is greater than consumption - which was shown through a higher concentration of oxyhemoglobin and decreased concentration of deoxyhemoglobin [17].

\subsection{Muscle Oxygenation Procedures}

During both Halo and sham 10km time trials, a Moxy NIRS monitor (Fortiori Design, Minnesota, USA) was placed on the dominant legs' vastus lateralis (VL) - distal part of the VL muscle belly (10-15 cm above the proximal border of the patella) [18]. The monitor was attached following cleaning with an alcohol wipe and secured with a double-sided adhesive disk and covered by a dark athletic tape to reduce intrusion of light [19]. The Moxy monitor position on the participant's skin was marked to ensure the monitor was placed on the same site in the following testing session. A moving 5-second average was applied on the raw muscle $\mathrm{O}_{2}$ saturation $\left(\mathrm{SmO}_{2}\right)$ signal to reduce the noise created by movement [20]. During exercise, $\mathrm{SmO}_{2}$ represents the balance between $\mathrm{O}_{2}$ delivery and $\mathrm{O}_{2}$ extraction by the muscle [21]. Minimum and maximum $\mathrm{SmO}_{2}$ are the absolute lowest and highest 5-second average $\mathrm{SmO}_{2}$ reached during either of the $10 \mathrm{~km}$ time trial sessions (Halo or sham). Minimum and maximum $\mathrm{SmO}_{2}$ values were determined from the lowest and highest observed values throughout the entire Halo or sham time trials for each participant. All $\mathrm{SmO}_{2}$ values were normalized, so that 0 and $100 \%$ represent these minimum and maximum $\mathrm{SmO}_{2}$ of the participant, respectively. $\mathrm{SmO}_{2}$ values are presented in these normalized values in the results section of the main manuscript. McManus et al. [22] provided evidence that in this subject group, both Moxy and PortaMon produce physiologically credible tissue oxygen saturation index measures during rest and exercise.

\subsection{Statistical Analysis}

In previous research studies in which the Halo Sport device was used to enhance exercise performance in healthy adults, researchers reported significant results $(p<0.05)$ with a total of 9 [7] and 12 participants [14] in crossover studies with repeated measures design. Therefore, we aimed to include a larger sample than those described in these previous studies [10, 23-24] to ensure accurate analysis of the effects of the Halo Sport intervention. All results are expressed as means \pm standard deviation. Brain and muscle $\left(\mathrm{SmO}_{2}\right)$ oxygenation changes from baseline within each time trial (Halo and sham) were analyzed using data from the LPFC (fNIRS channels 1-4 averaged) and RPFC (fNIRS channels 5-8 averaged) and VL muscle, respectively. Paired t-tests were used to compare Halo and sham $10 \mathrm{~km}$ time to completion, overall RPE, immediately post-exercise blood lactate, $\mathrm{SmO}_{2}$ and $\mathrm{PFC}$ regions (left and right) for $\mathrm{O}_{2} \mathrm{Hb}$ and $\mathrm{tHb}$. Data was analyzed using GraphPad Prism 9.0.2.

\section{Results}

\subsection{Time to Completion}

A t-test revealed that there was no significant difference $(p=0.92)$ in time to complete a $10 \mathrm{~km}$ cycling time trial between Halo and sham conditions (17.58 $\pm 1.88 \mathrm{~min}$; $17.68 \pm 1.92 \mathrm{~min}$ ) (Table 3).

\subsection{Prefrontal Cortex Oxygenation}

As shown in Figure 2, t-tests were used to compare oxygenated hemoglobin $\left(\mathrm{O}_{2} \mathrm{Hb}\right)$ and total hemoglobin (tHB) in the right prefrontal cortex (RPFC) and the left prefrontal 
cortex (LPFC) between Halo and sham conditions. Results showed no significant difference $(p=0.70)$ in average RPFC oxygenation $(26.85 \pm 24.10 \mu \mathrm{mol}, 23.18 \pm 17.96 \mu \mathrm{mol})$ and no significant difference $(p=0.98)$ in average LPFC oxygenation $(13.53 \pm 3.84 \mu \mathrm{mol}, 13.56$ $\pm 4.18 \mu \mathrm{mol})$ between conditions. Additionally, results showed no significant difference $(p=0.86)$ in average LPFC tHB $(18.63 \pm 5.17 \mu \mathrm{mol}, 18.23 \pm 5.44 \mu \mathrm{mol})$ and no significant difference $(p=0.73)$ in average RPFC tHB $(40.87 \pm 39.86 \mu \mathrm{mol}, 35.46 \pm 30.65 \mu \mathrm{mol})$ between conditions.
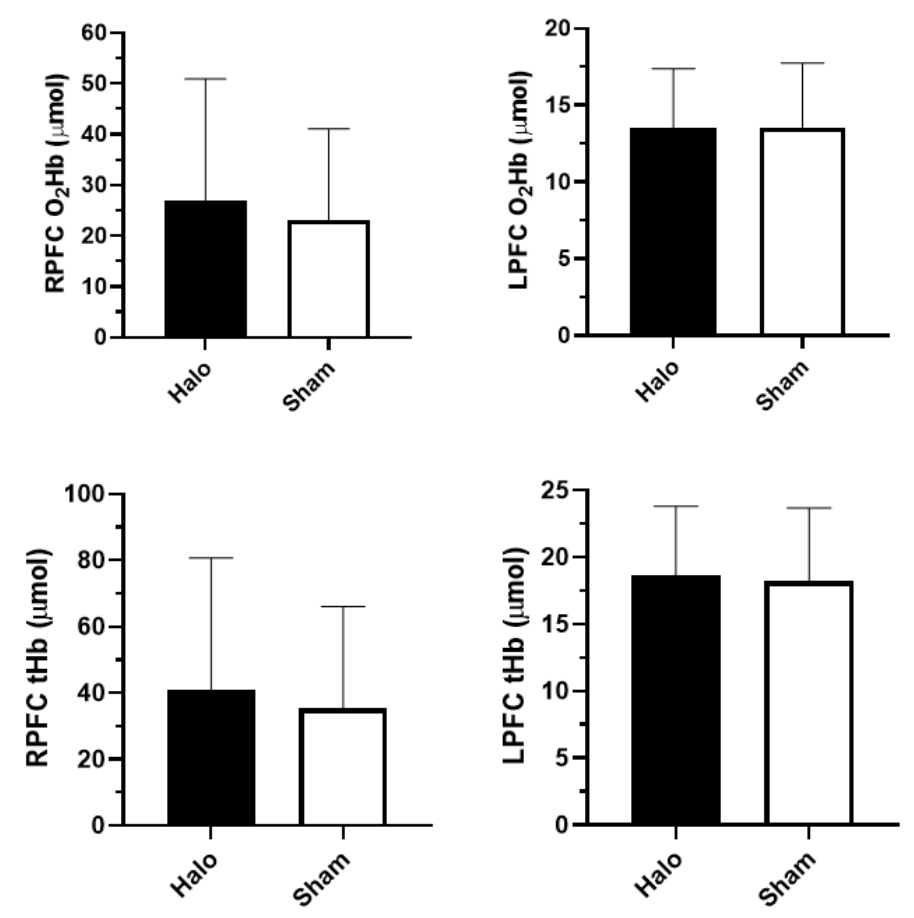

Figure 2. Brain Oxygenation Results.

\subsection{Muscle Oxygenation}

A t-test revealed that there was no significant difference $(p=0.40)$ in average muscle oxygenation between Halo and sham conditions, shown in Figure 3 below (18 \pm 9 percent, $23 \pm 15$ percent).

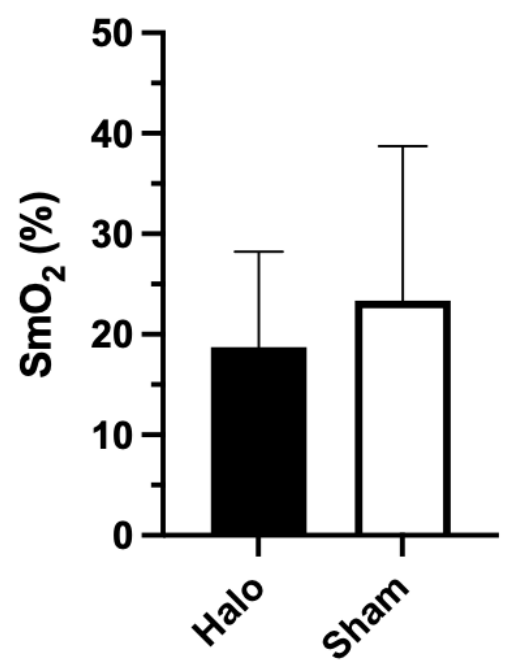

Figure 3. Muscle Oxygenation Results. 


\subsection{Heart Rate, Blood Lactate and Rating of Perceived Exertion}

Despite the application of tDCS, average HR did not show a significant difference $(p=0.74)$ compared to sham conditions $(163.5 \pm 17.2 \mathrm{bpm}, 160.9 \pm 20.1 \mathrm{bpm})$. There was no significant difference ( $p=0.78)$ observed in overall RPE between Halo and sham conditions $(16.20 \pm 1.99,15.95 \pm 2.03)$. Lastly, we analyzed the effects of tDCS on post-exercise BLa- levels and found no significant difference $(p=0.56)$ between Halo and sham conditions $(10.66 \pm 2.47 \mathrm{mmol} / \mathrm{L}, 9.99 \pm 2.88 \mathrm{mmol} / \mathrm{L})$. These results are displayed in Table 2 below.

Table 2. Results of Halo and sham on BLa', RPE, HR and Time.

\begin{tabular}{c|c|c}
\hline Characteristic & Halo & sham \\
\hline Bla $^{-}[\mathrm{mmol} / \mathrm{L}]$ & $10.66 \pm 2.46$ & $9.98 \pm 2.88$ \\
\hline RPE & $16.20 \pm 1.99$ & $15.95 \pm 2.03$ \\
\hline HR $[\mathrm{bpm}]$ & $163.5 \pm 17.2$ & $160.9 \pm 20.1$ \\
\hline Time $[\mathrm{min}]$ & $17.58 \pm 1.88$ & $17.67 \pm 1.92$ \\
\hline
\end{tabular}

Data are presented as means \pm standard deviations. $\mathrm{Mmol} / \mathrm{L}=$ millimoles per liter, $\mathrm{bpm}=$ beats per minute, $\min =$ minutes.

\section{Discussion}

The major finding in this study was that $10 \mathrm{~km}$ cycling time trial performance was unaffected by tDCS via the Halo Sport device. Results confirm some previous findings showing that $\mathrm{tDCS}$ has minimal effect on exercise performance and related physiological parameters [8, 23-25]. In addition, it was shown that both PFC and muscle oxygenation of the vastus lateralis were maintained over the entire portion of this self-paced, $10 \mathrm{~km}$ time trial. Moreover, brain and muscle oxygenation show similar trends, whereby PFC and $\mathrm{SmO}_{2}$ are well maintained and do not hinder or help self-paced exercise. That said, this study provides novel evidence to suggest that PFC oxygenation and $\mathrm{SmO}_{2}$ are well preserved during time trial cycling following Halo Sport administration when the intensity of exercise is free to vary in response to external and internal physiological cues.

Interest in the potential ergogenic effect of non-invasive brain stimulation has grown in the recent years and although there are various studies using tDCS and exercises, there are few focusing specifically on the Halo Sport device. Initial research focuses on single-joint isometric exercises, however whole-body exercise better simulates actual sporting competition. Therefore, whole-body exercise may be a more accurate method for assessing the ergogenic effects of tDCS on exercise performance. Of those studies done on whole-body exercise, results are inconsistent. Park et al. [14] found increased time to exhaustion during a constant load treadmill test after tDCS $(21.18 \pm 7.13$ mins; $18.44 \pm 6.32$ mins; $p=0.01$ ). Similarly, Vitor-Costa et al. [26] also saw increased time to exhaustion during an incremental cycling test, with no other significant results in other parameters (HR, RPE, Power output). However, this study found no significant difference in time to complete a $10 \mathrm{~km}$ cycling time trial. Our results are similar to those of Barwood et al. [23] who observed no changes in performance measures (time to exhaustion or power output) during a 20k time-trial following anodal tDCS (time to completion: $36 \mathrm{~min} 21 \mathrm{sec} \pm 52 \mathrm{sec}$ in sham, $36 \mathrm{~min} 21 \mathrm{sec} \pm 88 \mathrm{sec}$ in Halo; power output: 197 $\pm 12 \mathrm{~W}$ in sham, $197 \pm 20 \mathrm{~W}$ in Halo). Additionally, Angius et al. [5] found no significant difference $(p=0.06)$ in a time to exhaustion task between tDCS and sham conditions $(16.58 \pm 8.49 \mathrm{~min}, 14.68 \pm 8.62 \mathrm{~min})$. One possible explanation for the ineffectiveness of tDCS on sport performance may be that results are dependent on the experimental environment, duration and intensity of stimulation, and electrode configuration or placement on the head. Additionally, Halo Sport is a commercially made tDCS device that allows minimal adjustments to these factors [7]. 
To the author's knowledge, no studies have investigated the relationship between tDCS, PFC oxygenation, and whole-body self-paced exercise. This study found an increase in activation from baseline but no changes in average left and right $\mathrm{PFC} \mathrm{O}_{2} \mathrm{Hb}_{\text {or }}$ tHB and no related time-trial performance changes between Halo and sham conditions. There is evidence that submaximal aerobic exercise increases activation of the PFC, as suggested by increases in $\mathrm{O}_{2} \mathrm{Hb}$ and $\mathrm{tHb}$ saturation [27]. Therefore, oxygenation of the PFC seems to be related to the intensity of exercise, when compared to the baseline period. Nonetheless, the effect of Halo Sport on any intensity parameters or cortical oxygenation remains unclear. Our results are similar to those of Muthalib et al. [28] who found no changes in levels of PFC activation with the application of anodal tDCS over the right motor cortex and no significant changes in an isometric contraction task of the elbow flexors. Additionally, Holgado et al. [29] found that 20-min of tDCS over the LPFC does not affect HR, RPE, or EEG during a 20-min self-paced time-trial in male cyclists. It is possible that through repetition and experience, the act of self-pacing becomes more automatic and requires less thought and less activation in the area of the brain used for processing.

Similarly, no studies have investigated the effects of transcranial direct current stimulation on muscle oxygen saturation. This study found similar values for average muscle oxygen saturation between Halo and sham conditions. As expected, muscle saturation decreased progressively throughout the time-trial task and recovered quickly after the cessation of exercise. These responses of muscle oxygen saturation during wholebody exercise have been well documented [27, 30]. Belardinelli et al. [30] observed that oxygen saturation in the vastus lateralis decreased progressively during an incremental $\mathrm{VO}_{2}$ max test. They further observed that $\mathrm{SmO}_{2}$ decreased rapidly during a medium work rate range and leveled off when nearing $\mathrm{VO}_{2}$ max or very hard work rates. Rupp and Perrey [27] noted similar results in local muscle oxygen saturation in the vastus lateralis during a maximal time to exhaustion cycling test. In tandem, these results suggest that during moderate and hard intensities of exercise there is a discrepancy between local muscle $\mathrm{O}_{2}$ delivery and utilization. Rupp and Perrey (2007) further observed an increase in deoxygenated hemoglobin $(\mathrm{HHb})$ which may indicate a limitation in delivery of $\mathrm{O}_{2}$ to the working muscles rather than an inability to utilize available $\mathrm{O}_{2}$.

Our study also found no effect on cardiorespiratory (HR) or physiological (Bla-) response during the $10 \mathrm{~km}$ time trial with the application of tDCS. These results are similar to those of Park et al. [14] who found no change in HR responses during a constant load treadmill test after 20 minutes of Halo stimulation. Additionally, there are various studies that found no changes in HR responses during different exercises tasks following tDCS $[8,23,26]$. However, results of studies investigating tDCS and exercise related cardiovascular responses are conflicting. Okano et al. [10] saw a decreased HR trend following anodal tDCS during an incremental exercise test. The autonomic nervous system and cardiac responses are regulated by both sympathetic and parasympathetic neural pathways. Parasympathetic pathways are said to regulate HR during rest and exercise at lower intensities, whereas at higher intensities, HR becomes controlled by sympathetic pathways. This may indicate that the intensity of a $10 \mathrm{~km}$ time-trial at full effort triggered a mostly sympathetic HR response, which may be unaffected by tDCS. Also, previous studies have reported no effect of tDCS on HR during exercise intensities that are near $\mathrm{VO}_{2}$ max [23]. This study further found no significant difference in post-exercise blood lactate levels between Halo and sham conditions. Similarly, Barwood et al. [23] found similar blood lactate results following a 20km time trial after tDCS $(9.96 \pm 3.29 \mathrm{mmol} / \mathrm{L}$, $8.08 \pm 3.21 \mathrm{mmol} / \mathrm{L})$. Although this study observed no significant difference in levels of blood lactate between Halo and sham conditions, these results indicate that this $10 \mathrm{~km}$ time trial was a high intensity task. 
Results of studies investigating tDCS and RPE are inconsistent. Okano et al. [10] found RPE to increase more slowly following anodal tDCS stimulation over the temporal cortex. However, our study showed no difference in RPE after stimulation. Barwood et al. [23] and Vitor-Costa et al. [26] found similar results for RPE during time to exhaustion cycling tasks after tDCS. These results may indicate that the motor cortex is not related to perceptual responses, rather perceptions of effort may be regulated by different parts of the brain (e.g. insular cortex, thalamus). Further, Angius et al. [25] found no changes in perceptions of pain in a time to failure task following anodal stimulation of the motor cortex. Many factors have been proposed as to why perceptions of effort and pain may be insensitive to the analgesic effects of tDCS such as attentional focus, release of endogenous opioids or catecholamines, and supraspinal inhibitory mechanisms.

There are several limitations to the current study that should be noted. Several studies using tDCS to target specific areas of the brain have shown promise, however, Halo Sport is a commercially made device that does not allow for many adjustments. Due to anatomical differences between participants, it is possible that stimulation via Halo Sport may have not targeted the motor cortex and may have influenced other areas of the brain. Additionally, this study focused on recreationally trained males, ages 18-45. Our results may not translate to other populations as tDCS may have different effects on more novice or elite individuals, females, or different age groups. Lastly, this study used a $10 \mathrm{~km}$ time trial to measure performance. It is possible that $\mathrm{tDCS}$ would produce different results with different modes of exercise or different distances.

\section{Conclusions}

The results of this study indicate that the application of tDCS through Halo Sport has no effect on $10 \mathrm{~km}$ time trial performance and related physiological parameters in recreationally trained male cyclists. Future research done using tDCS should examine the potential long-term impact of training using tDCS on exercise performance, the potential benefits of $\mathrm{tDCS}$ on different modes and intensities exercise, and the potential differences between different populations (e.g. females, younger/older adults). Additionally, the impact of tDCS on decision making and accuracy during sport situations may be of interest.

Author Contributions: Conceptualization and preparation of study materials, T.M. and A.A.; methodology, T.M., J.R., A.A. and F.D.; statistical analysis, T.M. and A.A.; writing-original draft preparation, T.M. and A.A.; writing - critical review, editing and composition of certain portions, K.C., J.R. and F.D. ; supervision, T.M. All authors have read and agreed to the published version of the manuscript.

Funding: This research received no external funding.

Institutional Review Board Statement: The study was conducted according to the guidelines of the Declaration of Helsinki, and approved by the Institutional Review Board (or Ethics Committee) of the University of Northern Iowa (protocol code 20-0027 and 6 October 2020).

Informed Consent Statement: Informed consent was obtained from all subjects involved in the study.

Data Availability Statement: The group data presented in this study are available on request from the corresponding author. The individual data are not publicly available due to privacy and confidentiality.

Conflicts of Interest: The authors declare no conflict of interest. 
1. Amann, M. Central and Peripheral Fatigue: Interaction during Cycling Exercise in Humans. Med. Sci. Sports Exerc. 2011, 43 (11), 2039-2045. https://doi.org/10.1249/MSS.0b013e31821f59ab.

2. Neyroud, D.; Vallotton, A.; Millet, G. Y.; Kayser, B.; Place, N. The Effect of Muscle Fatigue on Stimulus Intensity Requirements for Central and Peripheral Fatigue Quantification. Eur. J. Appl. Physiol. 2014, 114 (1), $205-215$. https://doi.org/10.1007/s00421-013-2760-2.

3. Schillings, M. L.; Hoefsloot, W.; Stegeman, D. F.; Zwarts, M. J. Relative Contributions of Central and Peripheral Factors to Fatigue during a Maximal Sustained Effort. Eur. J. Appl. Physiol. 2003, 90 (5-6), 562-568. https://doi.org/10.1007/s00421-0030913-4.

4. Taylor, J. L.; Amann, M.; Duchateau, J.; Meeusen, R.; Rice, C. L. Neural Contributions to Muscle Fatigue: From the Brain to the Muscle and Back Again. Med. Sci. Sports Exerc. 2016, 48 (11), 2294.

5. Angius, L.; Hopker, J. G.; Marcora, S. M.; Mauger, A. R. The Effect of Transcranial Direct Current Stimulation of the Motor Cortex on Exercise-Induced Pain. Eur. J. Appl. Physiol. 2015, 115 (11), 2311-2319. https://doi.org/10.1007/s00421-015-3212-y.

6. Rossini, P. M.; Burke, D.; Chen, R.; Cohen, L. G.; Daskalakis, Z.; Di Iorio, R.; Di Lazzaro, V.; Ferreri, F.; Fitzgerald, P. B.; George, M. S.; Hallett, M.; Lefaucheur, J. P.; Langguth, B.; Matsumoto, H.; Miniussi, C.; Nitsche, M. A.; Pascual-Leone, A.; Paulus, W.; Rossi, S.; Rothwell, J. C.; Siebner, H. R.; Ugawa, Y.; Walsh, V.; Ziemann, U. Non-Invasive Electrical and Magnetic Stimulation of the Brain, Spinal Cord, Roots and Peripheral Nerves: Basic Principles and Procedures for Routine Clinical and Research Application: An Updated Report from an I.F.C.N. Committee. Clin. Neurophysiol. 2015, 126 (6), $1071-1107$. https://doi.org/10.1016/j.clinph.2015.02.001.

7. Huang, L.; Deng, Y.; Zheng, X.; Liu, Y. Transcranial Direct Current Stimulation with Halo Sport Enhances Repeated Sprint Cycling and Cognitive Performance. Front. Physiol. 2019, 10 (FEB), 1-7. https://doi.org/10.3389/fphys.2019.00118.

8. Baldari, C.; Buzzachera, C. F.; Vitor-Costa, M.; Gabardo, J. M.; Bernardes, A. G.; Altimari, L. R.; Guidetti, L. Effects of Transcranial Direct Current Stimulation on Psychophysiological Responses to Maximal Incremental Exercise Test in Recreational Endurance Runners. Front. Psychol. 2018, 9 (OCT), 1-10. https://doi.org/10.3389/fpsyg.2018.01867.

9. Mesquita, P. H. C.; Lage, G. M.; Franchini, E.; Romano-Silva, M. A.; Albuquerque, M. R. Bi-Hemispheric Anodal Transcranial Direct Current Stimulation Worsens Taekwondo-Related Performance. Hum. Mov. Sci. 2019, 66 (March), $578-586$. https://doi.org/10.1016/j.humov.2019.06.003.

10. Okano, A. H.; Fontes, E. B.; Montenegro, R. A.; De Tarso Veras Farinatti, P.; Cyrino, E. S.; Li, L. M.; Bikson, M.; Noakes, T. D. Brain Stimulation Modulates the Autonomic Nervous System, Rating of Perceived Exertion and Performance during Maximal Exercise. Br. J. Sports Med. 2015, 49 (18), 1213-1218. https://doi.org/10.1136/bjsports-2012-091658.

11. Rooks, C. R.; Thom, N. J.; McCully, K. K.; Dishman, R. K. Effects of Incremental Exercise on Cerebral Oxygenation Measured by Near-Infrared Spectroscopy: A Systematic Review. Prog. Neurobiol. 2010, $92 \quad$ (2), $134-150$. https://doi.org/https://doi.org/10.1016/j.pneurobio.2010.06.002.

12. Dietrich, A.; Sparling, P. B. Endurance Exercise Selectively Impairs Prefrontal-Dependent Cognition. Brain Cogn. 2004, 55 (3), 516-524. https://doi.org/10.1016/j.bandc.2004.03.002.

13. Fox, E. L. A Simple, Accurate Technique for Predicting Maximal Aerobic Power. J. Appl. Physiol. 1973, 35 (6), $914-916$.

14. Park, S. B.; Jun Sung, D.; Kim, B.; Kim, S. J.; Han, J. K. Transcranial Direct Current Stimulation of Motor Cortex Enhances Running Performance. PLoS One 2019, 14 (2), 1-11. https://doi.org/10.1371/journal.pone.0211902.

15. Smith, K. J.; Billaut, F. Influence of Cerebral and Muscle Oxygenation on Repeated-Sprint Ability. Eur. J. Appl. Physiol. 2010, 109 (5), 989-999. https://doi.org/10.1007/s00421-010-1444-4.

16. Orihuela-Espina, F.; Leff, D. R.; James, D. R. C.; Darzi, A. W.; Yang, G. Z. Quality Control and Assurance in Functional near Infrared Spectroscopy (FNIRS) Experimentation. Phys. Med. Biol. 2010, 55 (13), 3701-3724. https://doi.org/10.1088/00319155/55/13/009.

17. Herold, F.; Wiegel, P.; Scholkmann, F.; Müller, N. Applications of Functional Near-Infrared Spectroscopy (FNIRS) 
Neuroimaging in Exercise-Cognition Science: A Systematic, Methodology-Focused Review. J. Clin. Med. 2018,7 (12), 466. https://doi.org/10.3390/jcm7120466.

18. Billaut, F.; Buchheit, M. Repeated-sprint Performance and Vastus Lateralis Oxygenation: Effect of Limited O 2 Availability. Scand. J. Med. Sci. Sports 2013, 23 (3), e185-e193.

19. Paquette, M.; Bieuzen, F.; Billaut, F. Sustained Muscle Deoxygenation vs Sustained High VO2 during High-Intensity Interval Training in Sprint Canoe-Kayak. Front. Sport. Act. Living 2019, 1, 6.

20. Rodriguez, R. F.; Townsend, N. E.; Aughey, R. J.; Billaut, F. Influence of Averaging Method on Muscle Deoxygenation Interpretation during Repeated-sprint Exercise. Scand. J. Med. Sci. Sports 2018, 28 (11), 2263-2271.

21. Ferrari, M.; Muthalib, M.; Quaresima, V. The Use of Near-Infrared Spectroscopy in Understanding Skeletal Muscle Physiology: Recent Developments. Philos. Trans. R. Soc. A Math. Phys. Eng. Sci. 2011, 369 (1955), 4577-4590.

McManus, C. J.; Collison, J.; Cooper, C. E. Performance Comparison of the MOXY and PortaMon Near-Infrared Spectroscopy Muscle Oximeters at Rest and during Exercise. J. Biomed. Opt. 2018, 23 (1), 15007.

23. Barwood, M. J.; Butterworth, J.; Goodall, S.; House, J. R.; Laws, R.; Nowicky, A.; Corbett, J. The Effects of Direct Current Stimulation on Exercise Performance, Pacing and Perception in Temperate and Hot Environments. Brain Stimul. 2016, 9 (6), $842-849$.

24. Lattari, E.; de Oliveira, B. S.; Oliveira, B. R. R.; de Mello Pedreiro, R. C.; Machado, S.; Neto, G. A. M. Effects of Transcranial Direct Current Stimulation on Time Limit and Ratings of Perceived Exertion in Physically Active Women. Neurosci. Lett. 2018, 662, 12-16. https://doi.org/10.1016/j.neulet.2017.10.007.

25. Angius, L.; Mauger, A. R.; Hopker, J.; Pascual-Leone, A.; Santarnecchi, E.; Marcora, S. M. Bilateral Extracephalic Transcranial Direct Current Stimulation Improves Endurance Performance in Healthy Individuals. Brain Stimul. 2018,11 (1), $108-117$. https://doi.org/10.1016/j.brs.2017.09.017.

26. Vitor-Costa, M.; Okuno, N. M.; Bortolotti, H.; Bertollo, M.; Boggio, P. S.; Frengi, F.; Altimari, L. R. Improving Cycling Performance: Transcranial Direct Current Stimulation Increases Time to Exhaustion in Cycling. PLoS One 2015, 10 (12). https://doi.org/10.1109/ATEE.2015.7133822.

27. Thomas, R.; Stephane, P. Prefrontal Cortex Oxygenation and Neuromuscular Responses to Exhaustive Exercise. Eur. J. Appl. Physiol. 2008, 102 (2), 153-163.

28. Muthalib, M.; Kan, B.; Nosaka, K.; Perrey, S. Effects of Transcranial Direct Current Stimulation of the Motor Cortex on Prefrontal Cortex Activation during a Neuromuscular Fatigue Task: An FNIRS Study. In Oxygen Transport to Tissue XXXV; Springer, 2013; pp 73-79.

29. Holgado, D.; Zandonai, T.; Ciria, L. F.; Zabala, M.; Hopker, J.; Sanabria, D. Transcranial Direct Current Stimulation (TDCS) over the Left Prefrontal Cortex Does Not Affect Time-Trial Self-Paced Cycling Performance: Evidence from Oscillatory Brain Activity and Power Output. PLoS One 2019, 14 (2), e0210873.

30. Belardinelli, R.; Barstow, T. J.; Porszasz, J.; Wasserman, K. Changes in Skeletal Muscle Oxygenation during Incremental Exercise Measured with near Infrared Spectroscopy. / Modifications de 1' Oxygenation Du Muscle Squelettique Lors d ' Un Exercice Progressif, Mesurees Par Spectroscopie Infrarouge. Eur. J. Appl. Physiol. Occup. Physiol. 1995, 70 (6), $487-492$. 